\title{
Large-Displacement Vertical Microlens Scanner With Low Driving Voltage
}

\author{
Sunghoon Kwon, Student Member, IEEE, Veljko Milanovič, Member, IEEE, and Luke P. Lee
}

\begin{abstract}
We have designed, fabricated, and demonstrated large vertical displacement vertical microlens scanners with low $(<10 \mathrm{~V})$ driving voltage using silicon-on-insulator technology. The unique isolated and pre-engaged vertical comb-drive sets and the coupled-torsion flexure design provide both upward and downward piston motions, as well as low driving voltages. Single-directional devices demonstrate maximum static downward displacement of $8 \mu \mathrm{m}$ at $10 V_{\mathrm{dc}}$. Bidirectional devices demonstrate vertical actuation from -6.5 to $+9 \mu \mathrm{m}$ at $\max 12 V_{\mathrm{dc}}$, and a vertical displacement of up to $55 \mu \mathrm{m}$ peak-to-peak is achieved at the resonance near $400 \mathrm{~Hz}$. The lens motion shows piston motion with a small tilt angle of less than $0.034^{\circ}$ and the compensation of the tilt using an isolated comb bank is demonstrated.
\end{abstract}

Index Terms-Integrated optics, microlens scanner, microoptics, microoptoelectromechanical systems, silicon-on-insulator, variable focus, vertical comb-drive.

\section{INTRODUCTION}

$\mathbf{T}$ HREE-DIMENSIONAL (3-D) raster scanning is a key component for a wide range of photonic applications. A miniaturized 3-D raster scanning module with low power and cost can be used in 3-D imaging microelectromechanical system (MEMS) microscopes [1], retinal displays [2], free-space optical communication networks with micromotes [3], and 3-D MEMS memory [4]. MEMS-based microphotonics provide promising solutions to implement miniaturized cost-effective 3-D raster scanning modules.

We have been developing a miniaturized 3-D raster-scanning module which can fit within $1 \mathrm{~mm}^{3}$ as a key part of a microconfocal imaging array ( $\mu \mathrm{CIA})$ for lab-on-a-chip applications [1]. As a first step, we have already fabricated a two-dimensional (2-D) transmissive raster scanner and successfully demonstrated in-plane scanning by stacking two independent laterally actuated microlens scanners [1]. Stacking a vertically actuated microlens scanner on the developed 2-D scanner will allow complete 3-D raster scanning.

Previously, vertical comb-drive actuators with limited vertical displacements have been reported using surface micromaching for phase modulator applications, and their maximum displacement was limited by the thickness of the polysilicon layers, which is usually less than a few micrometers [5], [6]. As a result, silicon-on-insulator (SOI) wafers with a significantly thicker device layer have become the preferred choice for attaining large vertical displacements [2], [7]. In particular, the staggered

Manuscript received April 22, 2002. revised July 3, 2002.

S. Kwon and L. P. Lee are with the Berkeley Sensor and Actuator Center Department of Bioengineering, University of California, Berkeley, CA 94720-1762 USA (e-mail: lplee@ socrates.berkeley.edu).

V. Milanović is with the Adriatic Research Institute, Berkeley, CA 947041029 USA

Digital Object Identifier 10.1109/LPT.2002.803331.

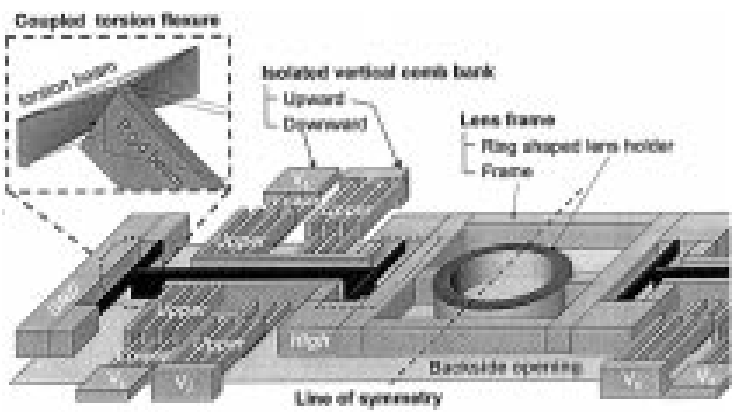

Fig. 1. Schematic of left-half side of the high aspect ratio vertical actuator based on isolated vertical comb-drives for bidirectional piston motion. All comb-banks are in an SOI layer.

comb-drive performed well for high-speed applications [2]. In terms of the static actuation, however, the staggered comb-drive is limited to unidirectional rotation only because all the lower comb-fingers are defined in substrate and electrically coupled. Bidirectional static actuation can be implemented by an electrically isolated vertical comb-drive, of which both lower and upper comb-fingers are defined in SOI as presented in this work. Also, pre-engaged vertical comb-drives are highly favorable to accomplish linear force control and lower driving voltages at a given spring constant for the static actuation. The pre-engagement length of twice the separation gap is required for the generation of the maximum constant force [6]. A previously reported implementation of the isolated and pre-engaged vertical comb-drives addresses the above objectives [8], but requires multi-substrate structure bonding and transfer with inherently limited alignment between comb-fingers. Lastly, the monolithic implementation of vertical actuators in a single-crystal silicon layer is desired for repeatability and reliability of the devices.

In this letter, we present the design, fabrication, and characterization of monolithic SOI vertical comb-drive actuators with integrated microlenses. Our pre-engaged and isolated vertical comb-banks defined in a monolithic SOI layer enable bidirectional, low voltage, large displacement, and linear actuation of the microlens, together with our unique coupled torsion flexures. This isolated comb-banks make it possible to compensate slight lens tilting due to variations in fabrication, as well as to actuate both upward and downward, thereby doubling the total static scanning range.

\section{DESIGN CONSIDERATIONS}

The four-mask process described in Section III provides four design layers with substantial flexibility: High, Lower, and Upper layers, and Backside opening, as depicted in Fig. 1. The thickness of the High layer is a full SOI device thickness of $50 \mu \mathrm{m}$. The Upper and Lower layers each have a thickness 
of $30 \mu \mathrm{m}$ and the overlap between the two layers is $10 \mu \mathrm{m}$. Our vertical scanners consist of three major units: a lens frame, coupled torsion flexures, and isolated comb banks. Fig. 1 shows the left-half side of the bidirectional device.

The lens frame consists of a ring-shaped lens holder for microlens integration and a frame for placing flexures and comb banks. The inner and outer diameters of the ring-shaped lens holder are design parameters related with required numerical aperture and entrance pupil size.

Two coupled torsion flexures are connected to both ends of the lens frame. A coupled torsion flexure is a set of two torsion beams in the Upper layer connected by a rigid beam in the High layer, as is shaded in Fig. 1. When the lens frame is actuated upward and downward by the electrostatic force generated by vertical comb drives, four torsion beams in the two coupled torsion flexures are not bent, but twisted by the rigid connecting beams, as shown in Fig. 1 (inset). This substitution of the difficult out-of-plane bending of high aspect ratio thick beams with more compliant torsion is an attractive flexure design, which maximize vertical compliance for low voltage actuation. According to our simulation, our coupled torsion flexure is 80 times more compliant than the double folded flexures [11] of the same length. In addition, small lateral bending of the upper torsion beams provides elongation needed in vertical displacement to prevent nonlinear increase in the vertical spring constant.

The isolated comb bank is a set of upper and lower comb-fingers defined in the Lower and Upper layer with $10 \mu \mathrm{m}$ of pre-engagement initial overlap between the upper and lower combfingers. Because both comb fingers are all in the SOI device layer, isolation between comb banks is achieved monolithically, which allows independent actuation between comb-fingers for bidirectional motion and tilting compensation. Fig. 1 shows the schematic of four isolated comb-drive sets with control voltages $V_{1}-V_{4}$. The right side of the line of symmetry has comb banks controlled by $V_{3}$ and $V_{4}$ also denoted in Fig. 3. The device is grounded through flexures. Normal upward motion is powered by $V_{2}$ and $V_{4}$, while downward motion is powered by $V_{1}$ and $V_{3}$. The unidirectional device is the same as bidirectional device, except its comb-banks are only for downward motion and they are doubled in number. Tilt compensation for extreme accuracy is possible with independent control of $V_{1}-V_{4}$. Applying an additional dc voltage to the other side of the line of symmetry can compensate the tilt in that line of the symmetry axis. To get large displacements at low driving voltages, we need to maximize the area of comb-banks at a given device size while minimizing in-plane rotational instability. This in-plane instability, which is determined by the ratio of the vertical stiffness to the in-plane rotational stiffness, limits the maximum extension of the comb-banks. Therefore, we placed comb-banks close to the coupled torsion flexures by placing the torsion beam inside the frame, as shown by the dashed box in Fig. 1.

\section{FABRICATION}

The four-mask fabrication methodology, which provides the above-mentioned three design layers and backside opening, was demonstrated previously [9]. Recently, the process has been improved to allow self-aligned positioning of those layers for implementation of vertical actuators [10]. The outline of this im-

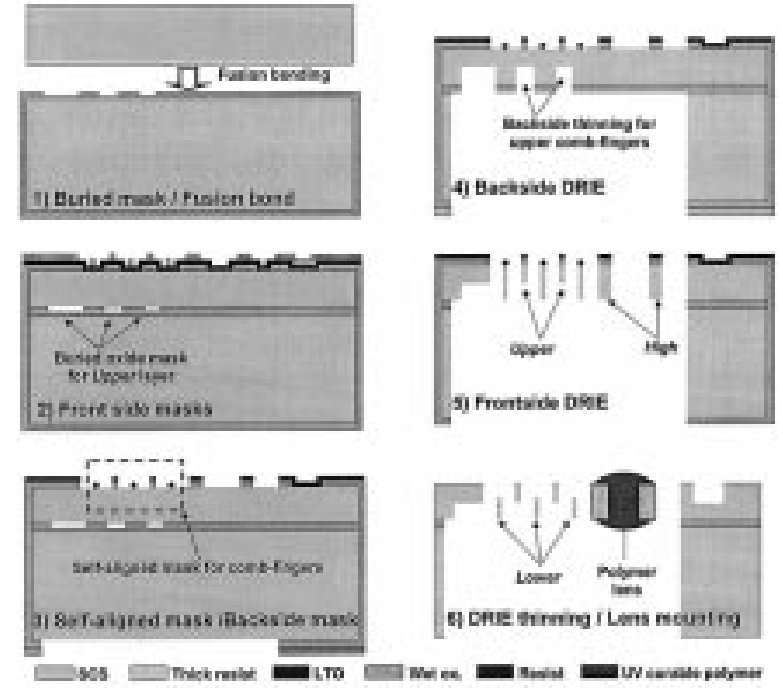

Fig. 2. Fabrication process for the vertical microlens scanner.

proved fabrication process is shown in Fig. 2 with the following steps.

1) A double-sided polished wafer of $300-\mu \mathrm{m}$ thickness with wet oxide is etched by the buried mask and then fusion bonded with another wafer. The resulting SOI wafer is ground and polished to desired device layer thickness of $50 \mu \mathrm{m}$, and oxide is stripped.

2) The remaining three front masks are imprinted in oxide films of different thickness before the first deep reactive ion etch (DRIE) as follows, in order to avoid lithography on etched wafer. After new thermal oxidation, first the front-side mask for the Upper comb-fingers and High rigid beams is patterned. This mask design is actually larger than the desired High and Upper to allow for the alignment margin in the next step. Then a layer of LPCVD oxide is deposited. The second front-side mask is then applied. This second mask has the actual device layers' dimensions and is used to pattern the LPCVD oxide, as well as the thicker thermal oxide underneath it. This effectively self-aligns all three device layers to a single photoresist mask.

3) The self-alignment etch for masks is performed with this second mask, resulting in an oxide layer with two thickness on the front side. This mask self-alignment in the mask level simplifies the previously demonstrated self-alignment method [7], which requires multiple DRIE steps. The backside of the wafer is patterned with thick resist, completing the preparation of all four masks for DRIE. This unique self-alignment method is described in more in detail in [10].

4) A large backside window is opened using DRIE, followed by a $20-\mu \mathrm{m}$ thinning of the Upper layer from the backside using the buried oxide mask, such that a 30- $\mu$ m thick Upper layer will result.

5) All three layers are defined by a front-side DRIE step through the entire device layer, followed by a RIE timed etch of the remaining thin LTO.

6) Lower is then thinned down to desired thickness by the final front-side DRIE, in our case down to $30 \mu \mathrm{m}$. Thus, selfaligned vertical comb-fingers with pre-engagement of $10 \mu \mathrm{m}$ are accomplished. 


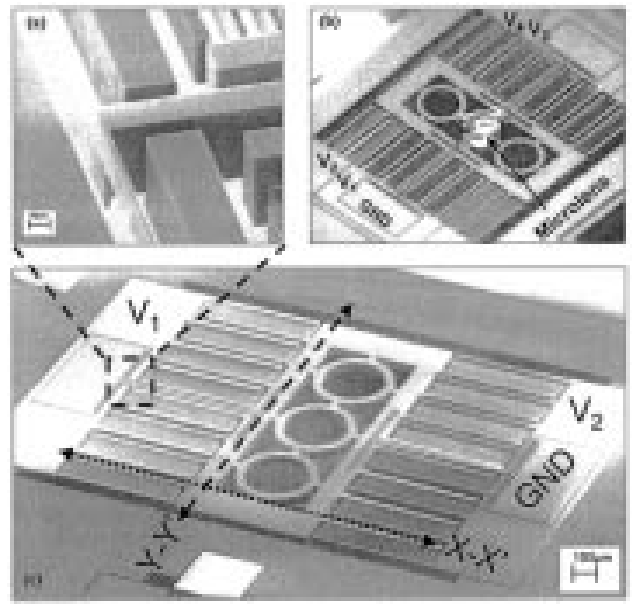

Fig. 3. SEM and picture for fabricated vertical microlens scanner: (a) SEM of zoomed coupled torsion flexure. (b) Microscopic picture of bidirectional device with polymer lens. (c) SEM of unidirectional device.

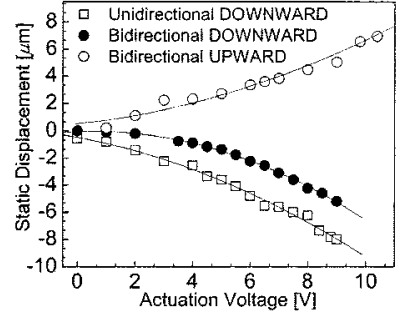

(a)

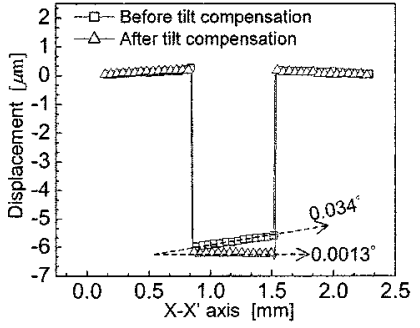

(b)
Fig. 4. (a) Static vertical displacement of the unidirectional and bidirectional devices. (b) Tilt compensation by asymmetric driving voltage. Tilt reduced from $0.034^{\circ}$ to $0.0013^{\circ}$ after compensation.

In addition to the benefits of this pre-engagement, discussed in the Introduction, it also reduces the risk of significant disengagement through possible faulty fabrication, such as overetching in this step. After cleaning of surface oxides and any polymer residue, the microlens is integrated. An ultraviolet (UV) curable polymer droplet is applied to the ring shape lens holder and the surface tension of the polymer forms a lens shape previously characterized in [1]. Exposure to UV light solidifies the polymer droplet on the 300- $\mu$ m-diameter lens holder. Fabricated unidirectional and bidirectional devices are shown in Fig. 3(b) and (c), respectively.

\section{RESULTS AND DISCUSSION}

Static and dynamic characteristics of the vertical microlens actuator were measured using a laser doppler vibrometer. Fig. 4(a) shows the static vertical displacement for both unidirectional and bidirectional vertical microlens scanners. The devices have large vertical displacements up to $\pm 10 \mu \mathrm{m}$ at sub-10-V driving voltages. This featured low-voltage actuation is due to compliant coupled torsion flexures, pre-engagement of vertical comb fingers, and high-density comb banks enabled by the buried design of the coupled flexure, which allows more area for comb drives. Bidirectional actuation enabled by isolated comb banks doubles the maximum possible displacement compared with unidirectional actuation. At resonance near $400 \mathrm{~Hz}$, the maximum displacement was $55 \mu \mathrm{m}$, and the scanning range control using linear amplitude modulation was measured.
Resonance frequency after microlens integration was measured to be $10 \%$ lower due to the added mass of the lens.

Any imperfections and asymmetries in the fabrication could result in undesired tilting of the lens plate during actuation. We characterized this aspect of our devices using an interferometric profiler. The maximum measured tilting during piston motion in the $X-X^{\prime}$ axis was only $0.034^{\circ}$ at $10 \mathrm{~V}$ and one tenth of that tilt in the $Y-Y^{\prime}$ axis. Pure piston motion with this negligible tilting angle is enough for most applications; however, compensation to zero tilt is also possible for applications requiring extreme accuracy by using asymmetric driving using our featured isolated comb banks. To demonstrate the compensation capability using the isolated comb banks, we tried to compensate the $X-X^{\prime}$ axis tilting by applying asymmetric voltage to either side of line of symmetry; i.e., by applying asymmetric voltage to $V_{1}$, and $V_{2}$, as indicated in Fig. 3(a). The compensation result is shown in Fig. 4(b) by acquiring a profile along with $X-X^{\prime}$ axis. The rectangular-marked profile represents symmetric actuation with $10 \mathrm{~V}$ and the tilt of the lens frame was $0.034^{\circ}$. The tilt is compensated to $0.0013^{\circ}$ by applying asymmetric actuation voltages of 10 and $11.456 \mathrm{~V}$ to the electrodes V1 and V2 in Fig. 3, respectively. Theoretically, perfect dynamic tilting compensation is possible using this technique.

\section{CONCLUSION}

We have demonstrated low-voltage and large-static displacement vertical actuators with integrated microlenses on a high-aspect ratio SOI. Due to the buried coupled torsion flexure and isolated vertical comb banks with pre-engagement, large-displacement vertical piston motion of a microlens is demonstrated with a low driving voltage. This device is not only a key part of our continuing effort for $\mu \mathrm{CIA}$, but is also attractive for other photonic applications, including optical communications.

\section{REFERENCES}

[1] S. Kwon and L. P. Lee, "Stacked two dimensional microlens scanner for micro confocal imaging array," in Proc. 15th Annu. IEEE Int. MEMS 2002 Conf., Las Vegas, NV, 2002, pp. 483-486.

[2] R. Conant, J. Nee, K. Lau, and R. Muller, "A flat high-frequency scanning micromirror," in Proc. Solid-State Sensor and Actuator Workshop, Hilton Head, SC, June 2000, pp. 6-9.

[3] B. Warneke, M. Last, K. Liebowits, and K. S. J. Pister, "Smart dust: Communicating with a cubic-millimeter computer," Compueter Magazine, pp. 44-51, Jan. 2001.

[4] Y. Kawata, "Three-dimensional memory," in Proc. SPIE, vol. 4081, 2000, pp. 76-85.

[5] A. P. Lee, C. F. McConaghy, P. A. Krulevitch, E. W. Campbell, G. E. Sommargren, and J. C. Trevino, "Electrostatic comb drive for vertical actuation," in Proc. SPIE, vol. 3224, 1997, pp. 109-19.

[6] J.-L. A. Yeh, C.-Y. Hui, and N. C. Tien, "Electrostatic model for an asymmetric vertical combdrive," J. Microelect. Syst., vol. 9, no. 1, pp. 126-135, Mar. 2000.

[7] U. Krishnamoorthy and O. Solgaard, "Self-Aligned vertical comb-drive actuators for optical scanning micromirrors," in Proc. Optical MEMS'01, Okinawa, Japan, Sept. 2001, pp. 41-42.

[8] J. Lee, Y. Ko, B. Choi, J. Kim, and D. Jeon, "Linear actuation of silicon scanning mirror for laser display," in Proc. Optical MEMS'01, Okinawa, Japan, Sep. 2001, pp. 101-102.

[9] V. Milanović, "Multilevel beam SOI-MEMS for optical applications," in Proc. 9th IEEE Int. Conf. Electronics, Circuits, and Systems, Dubrovnik, Croatia, Sept. 2002, pp. 281-285.

[10] S. Kwon, V. Milanović, and L. P. Lee, "Vertical microlens scanner for 3D imaging," in Proc. Solid-State Sensor and Actuator Workshop, Hilton Head, SC, June 2002, pp. 227-230.

[11] W. C. Tang, T. H. Nguyen, M. W. Judy, and R. T. Howe, "Electrostatic-comb drive of lateral polysilicon resonators," Sens. Actuators, vol. A21-A23, pp. 328-331, 1990. 